\title{
Enhancement of Non-specific Resistance to Pseudomonas Pneumonia by a Synthetic Derivative of Muramoyl Dipeptide in Immunosuppressed Guinea Pigs
}

\author{
By YASUAKI OSADA, ${ }^{1}$ * TSUYOSHI OHTANI, ${ }^{1}$ TSUTOMU UNE, ${ }^{1}$ \\ HIDEMASA OGAWA ${ }^{1}$ AND KIKUO NOMOTO ${ }^{2}$ \\ ${ }^{1}$ Research Institute, Daiichi Seiyaku Co. Ltd, Edogawa-ku, Tokyo 134, Japan \\ ${ }^{2}$ Department of Microbiology and Immunology, School of Medicine, Kyushu University, \\ Higashi-ku, Fukuoka 812, Japan
}

(Received 29 May 1981; revised 27 November 1981)

\begin{abstract}
A synthetic derivative of muramoyl dipeptide, 6-O-stearoyl- $N$-acetylmuramoyl-L-alanyl-D-isoglutamine [L18-MDP(A)], showed a protective effect against bacteraemic and non-bacteraemic pneumonia caused by Pseudomonas aeruginosa in immunosuppressed guinea pigs. In about half of the animals treated with the compound before infection, death from bacteraemic pneumonia produced by intratracheal inoculation of $P$. aeruginosa was delayed for $7 \mathrm{~d}$, although all of the animals infected without prior treatment with the compound died within $4 \mathrm{~d}$ of infection. Multiplication of the organisms in the lung was also suppressed for at least $10 \mathrm{~d}$ by treatment with the compound when the animals inhaled an aerosol of $P$. aeruginosa. In contrast, in untreated animals the numbers of bacteria in the lung gradually increased from $10^{6}$ to $10^{9}$ c.f.u. $\mathrm{g}^{-1}$, and a few animals in which the organism increased to $10^{9}$ c.f.u. $\mathrm{g}^{-1}$ had died by 6 and $10 \mathrm{~d}$ after infection. In both healthy and immunosuppressed animals, the accumulation of polymorphonuclear leukocytes (PMNs) in a subcutaneous air-pouch injected with heat-killed organisms was augmented by subcutaneous treatment with L18-MDP(A) $1 \mathrm{~d}$ before bacterial injection. The phagocytic activity of peritoneal PMNs was also increased by treatment with this compound. The augmentation of protective mechanisms against pseudomonas pneumonia by L18-MDP(A) may be attributed at least partly to the increased chemotactic and phagocytic activity of PMNs.
\end{abstract}

\section{INTRODUCTION}

It has become apparent that whole cells of some bacterial species (Dubos \& Schaedler, 1957; Howard et al., 1959; Parant et al., 1975), as well as both extracted (Dubos \& Schaedler, 1957; Elin et al., 1976; Howard et al., 1959; Landy, 1956; Parant et al., 1975, 1977) and synthetic (Chedid et al., 1977; Parant et al., 1978) bacterial cell-wall components endow animals with nonspecific resistance to microbial infections. Muramoyl dipeptide has been recognized as the minimal structure required for bacterial peptidoglycan to show adjuvant activity for both antibody and cell-mediated response in animals (Ellouz et al., 1974). On the basis of these observations, the effects of many derivatives of muramoyl dipeptide on both bacterial infections and neoplastic diseases have been studied (Chedid et al., 1977; Parant et al., 1978). We have also found that a derivative of muramoyl dipeptide, 6-O-stearoyl- $\mathrm{N}$-acetylmuramoyl-L-alanyl-D-isoglutamine [L18-MDP(A)], stimulates resistance in mice to infection with a variety of Gramnegative and Gram-positive organisms (Matsumoto et al., 1981). Furthermore, it has been shown that this compound stimulates antibody formation, but not delayed-type hypersensitivity, to some protein antigens in mice (Azuma et al., 1976). These findings suggested that the increase in resistance to microbial infections of animals treated with L18-MDP(A) might in part be attributed to the increased activity of polymorphonuclear leukocytes (PMNs) against the pathogens, with or without involvement of circulating antibody. 
To test this hypothesis, we have studied the protective effect of L18-MDP(A) against bacteraemic and non-bacteraemic pneumonia.

\section{METHODS}

Muramoyl dipeptide. A synthetic derivative of muramoyl dipeptide, L18-MDP(A) (Fig. 1), was provided by $\mathrm{Dr}$ O. Nagase, Chemical Technology Research Center, Research Institute, Daiichi Seiyaku Co., Tokyo. It was dissolved in autoclaved Dulbecco's phosphate-buffered saline, pH 7.4 (PBS; Nissui Seiyaku Co., Tokyo) at a concentration of $500 \mu \mathrm{g} \mathrm{ml}^{-1}$ just before use.

Animals. Groups of 5-10 female Hartley guinea pigs weighing $250-300 \mathrm{~g}$ were housed in each cage and used after 1 week's quarantine for respiratory and gastro-intestinal disorders. Animals showing any symptoms of infection, such as coughing, snuffling or diarrhoea, or suffering from injuries were eliminated.

Bacteria. A virulent strain of Pseudomonas aeruginosa, strain 2126, was used in this study. It was isolated from a patient with chronic pyelonephritis in 1977 and maintained in a lyophilized state in our laboratory. It was cultivated overnight in Trypto-soy broth (Eiken Chemicals Co., Tokyo) at $37^{\circ} \mathrm{C}$. The broth culture was then diluted to $10^{-2}$ with fresh Trypto-soy broth to give approximately $10^{7}$ c.f.u. $\mathrm{ml}^{-1}$. This suspension was used as the inoculum for intratracheal infection.

Infection and treatment. The animals were inoculated subcutaneously with cortisone acetate (Corton; Banyu Pharmaceuticals Co., Tokyo) at a dose of $100 \mathrm{mg} \mathrm{kg}^{-1}$ daily for $4 \mathrm{~d}$ before infection. On the last day of cortisone

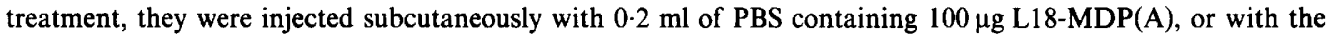
same volume of PBS alone as a control. After $24 \mathrm{~h}$, they were challenged with bacteria.

Bacteraemic pneumonia was established by direct inoculation of a $10^{-2}$ dilution of $P$. aeruginosa culture (see above) into the bronchotracheal tract. The cervical trachea of animals anaesthetized with pentobarbital (Nembutal; Dainippon Pharmaceuticals Co., Osaka) was surgically exposed and $0.2 \mathrm{ml}$ of the bacterial suspension was directly inoculated into the bronchotracheal tract toward the lung by means of a syringe with an L-shaped $26 \frac{1}{2}-$ gauge needle. The trachea was then briefly clamped, and the animal was positioned upright to assure penetration of organisms to the lower airway. The wound was then sutured, and the animals were allowed to stand in the normal position.

Non-bacteraemic pneumonia was produced by inhalation of an undiluted overnight culture of $P$. aeruginosa containing $10^{9} \mathrm{c}$.f.u. $\mathrm{ml}^{-1}$. Five animals were put in a plastic desiccator box $(31 \times 23 \times 26 \mathrm{~cm})$ equipped with a pocket nebulizer (Vaponefrine; USV Pharmaceuticals Co., New York) at the upper side and with an exhaust duct at the opposite lower side. Then $5 \mathrm{ml}$ of the culture was atomized through the nebulizer at a pressure of $2 \mathrm{kgf} \mathrm{cm}^{-2}$ for $10 \mathrm{~min}$ so that the number of organisms inhaled was approximately $10^{6} \mathrm{c.f.u} \cdot \mathrm{g}^{-1}$ of lung.

These animals were moved to another experimental room and given food and water ad libitum. The animals used for each infection were divided into two groups: one group was observed for mortality for 7-10 d, and the other was used for bacteriological examination of the blood and tissues.

Bacteriological examination. Animals challenged with organisms intratracheally were sacrificed at 3, 6, 10 and 24 $\mathrm{h}$ after challenge, and samples of blood, lung, spleen, liver and kidneys were removed aseptically. The blood samples collected by cardiac puncture and tissue homogenates were serially diluted 10-fold with PBS, and $0.1 \mathrm{ml}$ volumes of the dilutions were inoculated on to plates containing modified Drigalski agar (Eiken Chemicals Co., Tokyo). After overnight incubation at $37^{\circ} \mathrm{C}$ colonies were counted and the counts were converted into c.f.u. $\mathrm{ml}^{-1}$ or $\mathrm{g}^{-1}$. If necessary, the colonies of $P$. aeruginos $a$ were identified by conventional methods.

The aerosol-challenged animals were sacrificed at $1,3,6$ and $10 \mathrm{~d}$ after challenge, and the organisms in the blood and tissues were counted as described above.

Local migration of PMNs. Local migration of PMNs was tested in healthy and immunosuppressed guinea pigs according to a modification of the method of Ishikawa et al. (1968). Immunosuppression was produced by the same procedure as described above. The dorsal skin was treated with a depilator (Eba Cream; Tokyo Tanabe Pharmaceuticals Co., Tokyo), and $5 \mathrm{ml}$ of air was injected subcutaneously in the area of the dorsum scapulae to make an air-pouch. At the same time, the animals were injected subcutaneously with $100 \mu \mathrm{g}(0.2 \mathrm{ml})$ of L18MDP(A) in the lumbar area. After $24 \mathrm{~h}, 5 \mathrm{ml}$ of $2 \%$ (w/v) carboxymethylcellulose mixed with heat-killed organisms at a cell density of $10^{6}$ cells ml $^{-1}$ was injected into the air-pouch, and $0.2 \mathrm{ml}$ of the mixture containing tissue exudate was removed by a syringe with an 18-gauge needle at 2, 4, 6, 8 and $24 \mathrm{~h}$ after challenge; $0 \cdot 1 \mathrm{ml}$ volumes of exudate were then diluted to $10^{-1}$ with $0.05 \%(\mathrm{w} / \mathrm{v})$ Brilliant Cresyl Blue in $0.9 \%(\mathrm{w} / \mathrm{v})$ physiological saline, and vigorously mixed using a thermomixer at room temperature for $2 \mathrm{~min}$. The stained leukocytes were microscopically enumerated by use of a Fuchs-Rosenthal haemocytometer.

Phagocytic activity of PMNs. The test for phagocytic activity of PMNs was carried out with peritoneal cells induced by intraperitoneal injection of healthy and immunosuppressed animals with $20 \mathrm{ml}$ of $0.1 \%(\mathrm{w} / \mathrm{v})$ oyster glycogen (Daiichi Pure Chemicals Co., Tokyo) $20 \mathrm{~h}$ after subcutaneous treatment with $0.2 \mathrm{ml}$ of PBS containing L18-MDP(A) $(100 \mu \mathrm{g})$ or with the same volume of PBS. After $4 \mathrm{~h}$ each animal was challenged intraperitoneally 


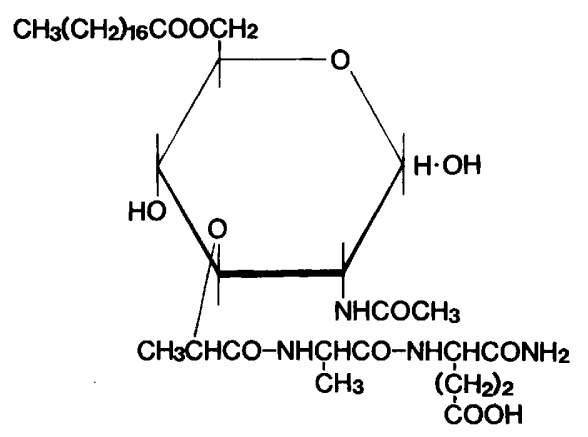

Fig. 1. Chemical structure of L18-MDP(A).

with $5 \mathrm{ml}$ of a suspension of live $P$. aeruginosa in Eagle's basal medium (Nissui Seiyaku Co., Tokyo) containing $2 \cdot 0$ $\times 10^{9}$ c.f.u. $\mathrm{ml}^{-1}$.

At 30,60 and $120 \mathrm{~min}$ after challenge, the peritoneal cells were harvested with $40 \mathrm{ml}$ of Eagle's basal medium supplemented with heparin $\left(100\right.$ units $\left.\mathrm{ml}^{-1}\right)$ and washed three times with Eagle's basal medium without heparin by centrifugation at $145 \mathrm{~g}$ for $10 \mathrm{~min}$. A drop of the washed sediment was swabbed on a glass slide, fixed with methanol, and stained with Giemsa solution. PMNs carrying bacilli within the cells were counted microscopically by scanning five continuous fields for each specimen. Phagocytic activity was expressed as the phagocytic index, and as the number of organisms engulfed per cell. The former was determined by examining $200 \mathrm{PMNs}$ at random and using the formula: phagocytic index $=100 \times($ No. of PMNs containing bacilli)/200. The number of organisms engulfed was estimated from the distribution pattern of cells according to the number of intracellular bacilli.

Significance test. Statistical analysis of the results was carried out by the use of (i) Fisher's test of direct probability (Ishii, 1975) and (ii) Fisher's test of least significant difference (Fryer, 1966). The first method was used to test the significance of the difference in incidence of bacterial recovery from the blood and tissues between L18$\operatorname{MDP}(A)$-treated animals and untreated controls. The second method was used to test the significance of the difference both in the number of $P$. aeruginosa in the lung, and in the number of PMNs which migrated to the site of bacterial infection, between both groups of animals. The statistical analysis of results of phagocytic activity of PMNs derived from L18-MDP(A)-treated and untreated animals was also carried out according to the second method. The error bars indicate \pm standard deviations (S.D.).

\section{RESULTS}

\section{Protective effect against bacteraemic pneumonia}

In the immunosuppressed animals challenged intratracheally, organisms were rapidly distributed from the pulmonary foci to other organs such as the spleen, liver and kidneys, probably via the blood or lymphatic system. Organisms were detected in the spleen of all these control animals $10 \mathrm{~h}$ after infection and were detected within $24 \mathrm{~h}$ in almost all of the other organs tested (Table 1). In contrast, by $10 \mathrm{~h}$ the organisms were detected in the spleen of only one of five immunosuppressed animals previously treated with L18-MDP(A). Organisms were detected in the spleen of seven out of eight animals $24 \mathrm{~h}$ after infection, while they were not detected in the liver or kidneys of five and six out of eight animals, respectively. Thus, the distribution of the organisms from the lung to other tissues was delayed by treatment with L18-MDP(A). This suggests that L18-MDP(A) may have enabled the animals to prevent the development of bacteraemia after pulmonary infection with a virulent strain of $P$. aeruginosa.

Almost all of the control immunosuppressed animals [i.e. those not given L18-MDP(A)] developed bacteraemia and seven out of ten had died by $24 \mathrm{~h}$ after infection. The three remaining animals had died by $4 \mathrm{~d}$ after infection (Fig. 2). In contrast, none of the animals treated with L18-MDP(A) died within $24 \mathrm{~h}$ after infection. On day 4, when all of the control animals had died, half of the treated animals were still alive, and four out of ten lived for at least $7 \mathrm{~d}$. 


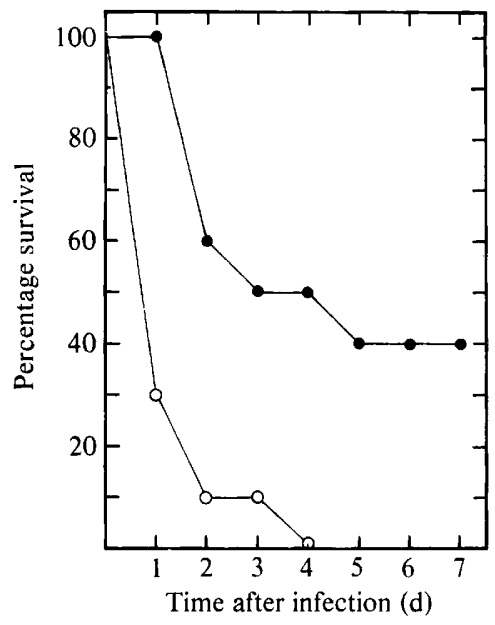

Fig. 2

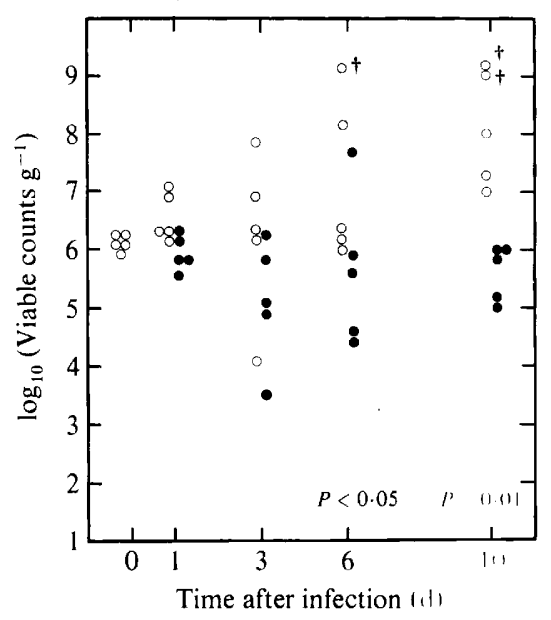

Fig. 3

Fig. 2. Protective effect of L18-MDP(A) against bacteraemic death in guinea pigs with $P$. aeruginosa pneumonia. Groups of ten animals treated with L18-MDP(A) (O) or untreated (O) were challenged intratracheally with a virulent strain of $P$. aeruginosa, as described in Methods, and observed for $7 \mathrm{~d}$ after infection. The results shown are from a single experiment.

Fig. 3. Suppressive effect of L18-MDP(A) on the growth of $P$. aeruginosa in the lungs of guinea pigs. The numbers of $P$. aeruginosa in the lungs of animals treated with L18-MDP(A) (O) or untreated (O) were determined by plate culture of homogenates of each lung. A few control animals in which organisms multiplied to $10^{9}$ c.f.u. $\mathrm{g}^{-1}$ in the lung had died $(\dagger)$ by days 6 and 10 after infection. On day 6 , the mean counts of organisms in the lung of test and control groups were $5.64 \pm 1.25$ and $7.24 \pm 1.42$, on the log scale, respectively (significantly different at the $5 \%$ level), and those on day 10 were $6 \cdot 19 \pm$ 1.19 and $8.12 \pm 1.02$, respectively (significantly different at the $5 \%$ and $1 \%$ level).

Table 1. Incidence of bacterial recovery from the blood and tissues of immunosuppressed animals challenged with $P$. aeruginosa

The results show, first, the number of animals whose visceral or blood cultures were positive for $P$. aeruginosa and, second, the number of animals tested, at the indicated times after infection.

\begin{tabular}{llllll}
\multirow{2}{*}{ Specimen } & & \multicolumn{4}{c}{ Incidence of bacterial recovery } \\
\multirow{2}{*}{ Lung } & Treatment & $3 \mathrm{~h}$ & $6 \mathrm{~h}$ & $10 \mathrm{~h}$ & $24 \mathrm{~h}$ \\
\multirow{4}{*}{ Spleen } & L18-MDP(A) & $5 / 5$ & $5 / 5$ & $5 / 5$ & $8 / 8$ \\
& PBS & $5 / 5$ & $5 / 5$ & $5 / 5$ & $8 / 8$ \\
Liver & L18-MDP(A) & $1 / 5$ & $1 / 5$ & $1 / 5^{*}$ & $7 / 8$ \\
& PBS & $1 / 5$ & $1 / 5$ & $5 / 5$ & $8 / 8$ \\
Kidneys & L18-MDP(A) & $0 / 5$ & $1 / 5$ & $1 / 5$ & $3 / 8$ \\
& PBS & $0 / 5$ & $1 / 5$ & $1 / 5$ & $7 / 8$ \\
Blood & L18-MDP(A) & $0 / 5$ & $0 / 5$ & $1 / 5$ & $2 / 8^{*}$ \\
& PBS & $0 / 5$ & $1 / 5$ & $1 / 5$ & $7 / 8$ \\
& L18-MDP(A) & $0 / 5$ & $0 / 5$ & $0 / 5$ & $1 / 8^{*}$ \\
& PBS & $1 / 5$ & $1 / 5$ & $1 / 5$ & $6 / 8$
\end{tabular}

* The incidence of bacterial recovery was significantly less than that in PBS controls at the $5 \%$ level.

Suppressive effect of $L 18-M D P(A)$ on growth of $P$. aeruginosa in the lung

By aerosol challenge with an overnight broth culture of $P$. aeruginosa, animals inhaled about $10^{6}$ c.f.u. $\mathrm{g}^{-1}$ of lung tissue. In the control animals, the number of organisms in the foci gradually increased from $10^{7}$ to $10^{9}$ c.f.u. $\mathrm{g}^{-1}$ (Fig. 3). Out of five animals, one and two in which the 


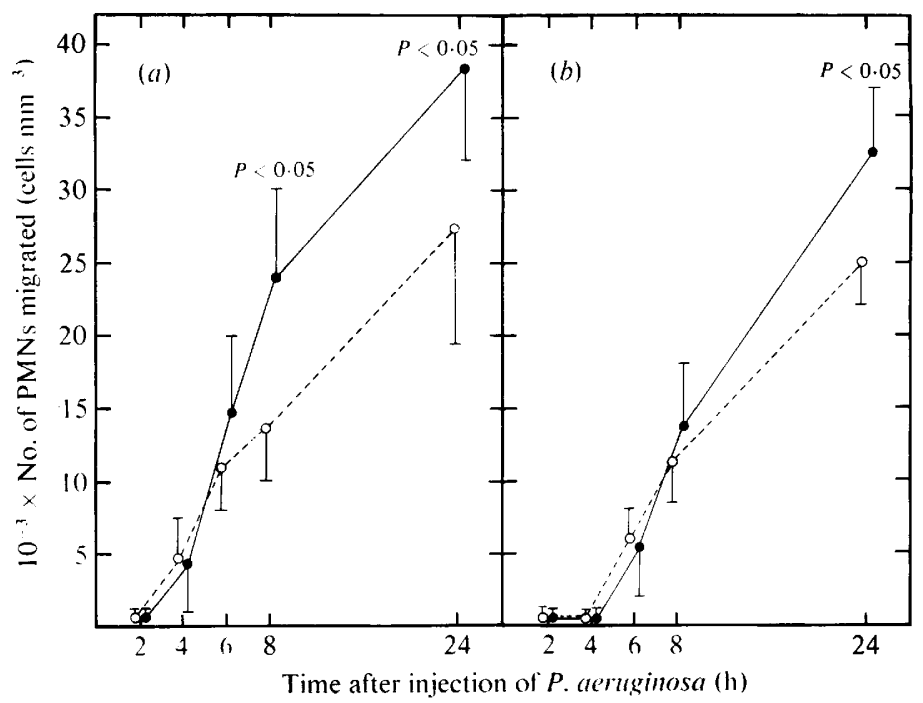

Fig. 4. Local migration of PMNs in healthy (a) and immunosuppressed $(b)$ guinea pigs. The local migration of PMNs was measured by the subcutaneous air-pouch technique, in which heat-killed $P$. aeruginosa were injected at a cell density of $10^{6}$ cells ml $^{-1} 1 \mathrm{~d}$ after animals were treated with L18MDP(A) (O) or without such treatment (O). The number of cells that migrated to the skin pouch was determined by microscopical quantification of smears of exudates removed from the pouches. Error bars indicate \pm S.D.

organisms increased to $10^{9}$ c.f.u. $\mathrm{g}^{-1}$ in the foci had died by days 6 and 10 after infection, respectively. None of the animals treated with L18-MDP(A) died, and the organisms that were localized in the lungs of these animals barely multiplied. Even on day 10, no increase in the number of organisms was observed. On days 6 and 10, the mean numbers of organisms in the foci of L18-MDP(A)-treated animals were $4.4 \times 10^{5}$ and $1.3 \times 10^{6}$ c.f.u. $\mathrm{g}^{-1}$, respectively. In contrast, those in the foci of untreated controls were $1.7 \times 10^{7}$ and $1.3 \times 10^{8}$ c.f.u. $\mathrm{g}^{-1}$, respectively. The differences in the bacterial counts in the foci between L18-MDP(A)-treated and untreated animals on days 6 and 10 were statistically significant at the $5 \%$ and $1 \%$ level, respectively. Furthermore, no organisms were recovered from the blood or other organs. Thus, L18-MDP(A) protected the animals against bacteraemia subsequent to the multiplication of organisms in the primary foci, and prevented distribution of the organisms to other organs, probably via the blood stream.

\section{Local response of PMNs}

The effect of L18-MDP(A) on the migratory response of PMNs to the site of injection of $\boldsymbol{P}$. aeruginosa in healthy and immunosuppressed animals was determined. Because of difficulties in analysing the migration of PMNs quantitatively in the lung, the skin pouch model devised as a screening method for anti-inflammatory agents (Ishikawa et al., 1968) was employed. This model can give highly reproducible results, providing the pouch is retained without haemorrhage. The number of PMNs in the pouches of healthy animals not treated with L18-MDP(A) increased linearly with time, and reached $27000 \mathrm{~mm}^{-3}$ by $24 \mathrm{~h}$ after challenge (Fig. $4 a$ ). In the animals treated with L18-MDP(A), on the other hand, the PMN counts increased logarithmically, and were significantly higher than those in control animals at 8 and $24 \mathrm{~h}$ after challenge $(P<0.05)$.

In immunosuppressed animals, there was a $2 \mathrm{~h}$ lag in the PMN response; then numbers increased linearly to $25000 \mathrm{~mm}^{-3} 24 \mathrm{~h}$ (Fig. 4b). The PMN counts in animals treated with L18MDP(A) also increased linearly with time, and were higher than those in control animals at 8 and $24 \mathrm{~h}$ after challenge. The difference in PMN counts between treated and untreated animals at $24 \mathrm{~h}$ was statistically significant at the $5 \%$ level. These findings indicate that the initial 


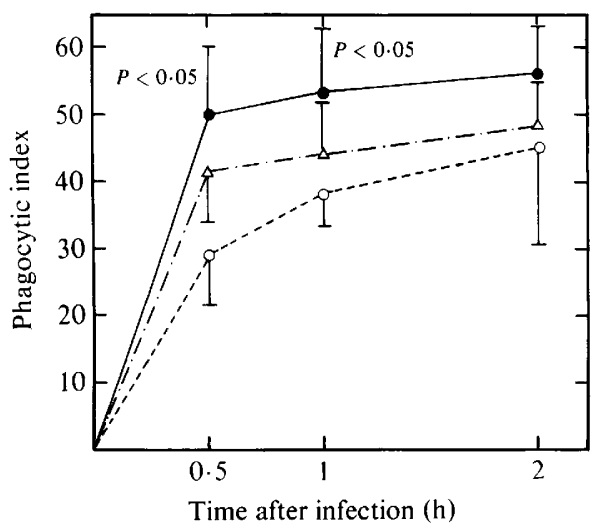

Fig. 5. Phagocytic indices of PMNs from healthy and immunosuppressed guinea pigs with or without treatment with L18-MDP(A). At each time after infection, groups of five healthy animals $(\triangle)$ and immunosuppressed animals treated with L18-MDP(A) $(O)$ or untreated $(O)$ were sacrificed and the peritoneal PMNs were harvested. The sediment of washed PMNs was smeared on a glass slide and the cells carrying organisms were enumerated microscopically after fixation and staining of the smears. The phagocytic index was calculated as indicated in Methods. The statistical analysis, by use of Fisher's test of least significant difference, of results for the healthy and adjuvant-treated/immunosuppressed groups was carried out against those for the untreated/immunosuppressed group. Error bars indicate \pm S.D.

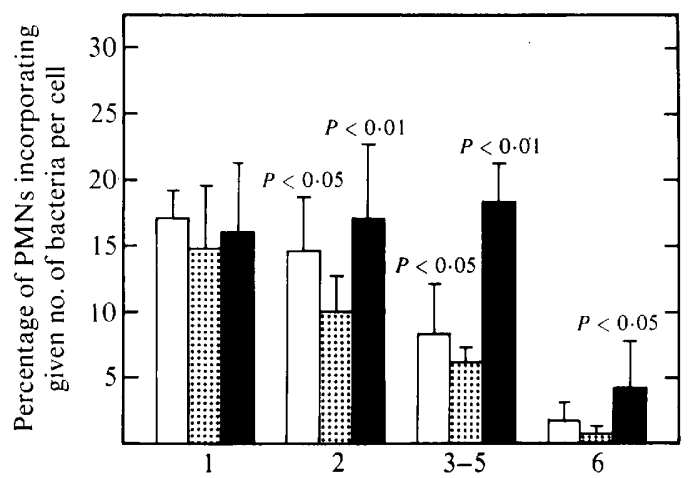

No. of intracellular bacteria

Fig. 6. Phagocytic activity of PMNs from healthy and immunosuppressed guinea pigs with or without treatment with L18-MDP(A). In the microscopical examination of the smears of PMN sediment, the number of organisms incorporated by each cell was determined, and the phagocytosed cells were scored according to the number of intracellular bacteria. The statistical analysis of results for the healthy ( $\square$ ) and adjuvant-treated/immunosuppressed groups $(\square)$ was performed against those for the untreated/immunosuppressed group ( $(\mathrm{E})$. The results shown were obtained $30 \mathrm{~min}$ after infection. The number of cells from treated animals which carried multiple organisms was significantly larger than that from untreated controls at the $1 \%$ or $5 \%$ level. Error bars indicate \pm s.D.

migration of PMNs toward the focus of injection was delayed by immunosuppression with cortisone acetate. This depressed response was, however, enhanced by treatment with L18$\operatorname{MDP}(\mathrm{A})$, and exceeded the response in untreated controls $24 \mathrm{~h}$ after bacterial challenge.

\section{Phagocytic activity}

The phagocytic activity of peritoneal PMNs induced by $0 \cdot 1 \%(\mathrm{w} / \mathrm{v})$ oyster glycogen was expressed as the phagocytic index and as the number of organisms engulfed per cell. The phagocytic indices of PMNs induced in untreated healthy animals, and in immunosuppressed animals with or without treatment with L18-MDP(A), are shown in Fig. 5. The index was always less in untreated immunosuppressed animals than in untreated healthy ones. Treatment 
with L18-MDP(A) $1 \mathrm{~d}$ before infection, however, restored the phagocytic capacity of PMNs in immunosuppressed animals. In this case, the phagocytic index exceeded that in healthy animals, though the difference was not statistically significant. The differences in phagocytic index between adjuvant-treated and untreated immunosuppressed animals at 30 and $60 \mathrm{~min}$ was statistically significant at the $5 \%$ level.

The distribution pattern of cells according to the number of intracellular bacteria at $30 \mathrm{~min}$ is illustrated in Fig. 6. As predicted by the phagocytic index, the capacity of PMNs to engulf bacteria was suppressed by cortisone treatment, this suppression being in turn reversed by treatment with L18-MDP(A). In immunosuppressed animals given L18-MDP(A), phagocytic activity exceeded that in untreated healthy animals. This could have been responsible for the elimination of larger number of organisms from the infection site seen after treatment with L18$\operatorname{MDP}(\mathrm{A})$.

\section{DISCUSSION}

Fatal bacteraemic pneumonia caused by $P$. aeruginosa was established by direct intratracheal inoculation of a broth culture of the bacterium into guinea pigs treated with cortisone acetate. The organisms were rapidly distributed to tissues other than the lung, probably via the blood or lymphatic system. Most of the animals died within $48 \mathrm{~h}$ after infection. This severe course of pseudomonas pneumonia may be attributed to depression of the host's defence by the treatment with cortisone acetate. Following the use of immunosuppressive agents such as glucocorticoid and cyclophosphamide, animals become depleted of phagocytes, particularly PMNs, the primary effector cells against bacterial invasion (Bicker et al., 1978; Dale et al., 1973, 1974; Peters et al., 1972). This PMN-inflammatory response was virtually absent or decreased in immunosuppressed guinea pigs (Pennington, 1977a) and patients (Sickles et al., 1975). Surgical invasion might act synergistically with cortisone treatment to promote immunosuppression. Without such an operation (i.e. aerosol challenge), the animals hardly ever developed bacteraemia, although organisms were localized in the lung for long periods of time and gradually proliferated there.

In fatal bacteraemic infection, L18-MDP(A) successfully protected the animals from death subsequent to the bacteraemic changes. The distribution of the organisms from the lung to other organs was delayed by the treatment. The suppressive effect of L18-MDP(A) on multiplication of the organisms in foci of infection was not surprising in the light of its stimulatory effect on phagocytic function. It has become evident that PMNs are primary effector cells in the elimination of a variety of micro-organisms from tissues. The significance of their phagocytic functions in protecting the host from microbial invasion has already been made clear by experiments in hosts whose phagocytic functions were depressed by X-irradiation (Gordee \& Simpson, 1967; Kaplan et al., 1952; Tatsukawa et al., 1979), treatment with cyclophosphamide (Bicker et al., 1978; Sharbaugh \& Grogan, 1969), vinblastin (Tripathy \& Mackaness, 1969) or vincristine (Saslaw et al., 1972). Furthermore, a correlation between decreased numbers of PMNs and risk from infection has been clearly shown (Bodey et al., 1966; Peters et al., 1972). If methods of augmenting the functions of PMNs can be devised, knowledge of the role of these cells in mechanisms of specific and/or non-specific resistance to microbial infection may be advanced extensively. The mode of action of L18-MDP(A) on the migration of PMNs also requires clarification.

Pennington (1977b) has devised a quantitative assay for pulmonary phagocytes. In his assay, however, the conditions of washing out the cells, and occasional leakage of lavaged fluid caused by lesions in the lung tissues, gave rise to substantial fluctuations in the results. Furthermore, the lavaged fluid contained not only PMNs but also a large number of pulmonary macrophages, and this complicated the results. In contrast, the subcutaneous air-pouch technique devised as a screening method for anti-inflammatory agents gave consistent results concerning the migratory response of PMNs. Although the anatomical and physiological features of this model are different from those of the lung, this assay may present enough evidence for analysis of the local response of PMNs against the bacterial invasion. By the use of this method, quantitatively reproducible results were obtained because of the direct removal of the migrated cells. 
In healthy animals, the number of PMNs migrating to the injection site increased linearly with time, and the response was further enhanced by treatment with L18-MDP(A). In immunosuppressed animals, however, the PMN response lagged a few hours after challenge, and gradually increased later. The delay in the PMN response may be attributed to the suppressive effect of cortisone acetate on the chemotaxis or mobilization of localized PMNs, as has been shown in other studies (Peters et al., 1972; Ward, 1966). Although treatment with L18-MDP(A) did not prevent the initial delay in PMN migration, the subsequent response was increased, eventually exceeding that in untreated controls $24 \mathrm{~h}$ after infection. This indicates that whereas L18-MDP(A) could not abrogate the suppressed chemotaxis of PMNs, it might restore the activity of blood leukocytes and/or bone marrow granulocyte precursors more rapidly.

Another function of PMNs, namely phagocytosis, was also enhanced by L18-MDP(A), irrespective of immunosuppression. In this connection, Pennington (1977a) also presented evidence to show little decrease in the phagocytic index, viability, and adherence of pulmonary alveolar macrophages derived from leukopenic guinea pigs continuously treated with cortisone acetate. The peritoneal PMNs from adjuvant-treated animals engulfed bacilli more vigorously than those from untreated animals. Statistically significant differences were observed between both groups of animals with regard to the phagocytosis of the organisms by PMNs which had migrated to the infection site. Thus, even in immunosuppressed animals, L18-MDP(A) could enhance their capacity to eliminate large numbers of bacteria localized in their tissues.

Buhles \& Shifrine (1977) demonstrated adjuvant-mediated protection against $P$. aeruginosa infection in a granulocytopenic model of mice produced by treatment with cyclophosphamide. This treatment produced not only granulocytopenia, but also monocytopenia and lymphopenia irrespective of adjuvant treatment. Nevertheless, the animals with adjuvant (BCG or Freund's Complete Adjuvant) were protected against subsequent challenge with $P$. aeruginosa. Buhles \& Shifrine (1977) attributed this protection to the activated macrophages on the basis of morphological criteria. They also described the finding that blood granulocytes and bone marrow progenitors recovered more rapidly from cyclophosphamide-induced myelosuppression when previously treated with BCG or Freund's Complete Adjuvant.

Pennington (1977b) emphasized that, even in immunosuppressed guinea pigs treated with cortisone acetate, PMNs were largely responsible for the elimination of the organisms from the foci. Tatsukawa et al. (1979) also found markedly increased mortality after the inoculation of a sublethal dose of $P$. aeruginosa in X-irradiated mice (regarded as PMN- and macrophagedepleted) but not in carageenan-treated mice (regarded as macrophage-depleted). From these observations, they concluded that macrophages, unlike PMNs, contributed little to the primary elimination of $P$. aeruginosa from the animal tissues. This situation has also been found in man (Young \& Armstrong, 1972).

We have placed emphasis on the PMN function as the first step in the analysis of the complex mechanisms of stimulation of resistance to bacterial infection following treatment with L18MDP(A). However, direct evidence concerning the mechanism of augmentation of PMN function by this compound is not available. Many investigators point to macrophage activation by muramoyl dipeptide derivatives. Therefore, in addition to the direct effect on PMNs, the possibility of the involvement of macrophages and/or lymphocytes in mechanisms of PMN activation by L18-MDP(A) should be studied further. Another possibility, namely that the compound may activate properdin systems clearly related to many humoral and subsequent cellular responses (Dukor et al., 1971; Gotze \& Muller-Eberhard, 1971), also remains to be studied further.

Protection against bacterial or fungal infections has significant clinical importance. The use of an immunostimulant for patients with granulocytopenia induced by cancer treatment, or with other neutropenic diseases, may well prove helpful in the control of opportunistic infections.

\section{REFERENCES}

Azuma, I., Sugimura, K., Taniyama, T., Yamawaki, M., Yamamura, Y., Kusumoto, S., OKada, S. \& Shibata, T. (1976). Adjuvant activity of myco- bacterial fractions: adjuvant activity of synthetic $\mathrm{N}$ acetylmuramyl dipeptide and the related compounds. Infection and Immunity 14, 18-27. 
Bicker, U., Hebold, G., Ziegler, A. E. \& Maus, W. (1978). Animal experiments on the compensation of the immunosuppressive action of cyclophosphamide by 2-[-2-cyanazyridinyl-(1)-]-2-[-2-carbamoylazyridinyl-(1)]-propane BM 12531. Experimental Pathology, Jena 15, 49-62.

Bodey, G. P., Buckley, M., Sathe, Y. S. \& Freirich, E. J. (1966). Quantitative relationships between circulating leukocyte and infection in patients with acute leukemia. Annals of Internal Medicine 64, 328340.

BUHles, W. C. \& Shifrine, M. (1977). Adjuvant protection against bacterial infection in granulocytopenic mice. Journal of Infectious Diseases 136, 90-95.

Chedid, L., Parant, M., Parant, F., Lefrancier, P., CHOAY, J. \& LEDERER, E. (1977). Enhancement of nonspecific immunity to Klebsiella pneumoniae infection by a synthetic immunoadjuvant ( $N$-acetylmuramyl-L-alanyl-D-isoglutamine) and several analogs. Proceedings of the National Academy of Sciences of the United States of America 74, 2089-2093.

Dale, D. C., Fauci, A. S. \& WolfF, S. M. (1973). The effect of cyclophosphamide on leukocyte kinetics and susceptibility to infection in patients with Wegener's granulomatosis. Arthritis and Rheumatism 16, 657-664.

Dale, D. C., Fauci, A. S. \& WolfF, S. M. (1974). Alternate-day prednisone: leukocyte kinetics and susceptibility to infections. New England Journal of Medicine 28, 1154-1158.

Dubos, R. J. \& Schaedler, R. W. (1957). Effects of cellular constituents of mycobacteria on the resistance to heterologous infections. I. Protective effect. Journal of Experimental Medicine 106, 703-717.

Dukor, P., Bianco, C. \& Nussenzweig, V. (1971). Bone marrow origin of complement-receptor lymphocytes. European Journal of Immunology 1, 491494.

Elin, R. J., WolfF, S. M. \& Chedid, L. (1976). Nonspecific resistance to infection induced in mice by a water-soluble adjuvant derived from $M$ ycobacterium smegmatis. Journal of Infectious Diseases 133, 500 505.

Ellouz, F., Adam, A., Cirbaru, R. \& Lederer, E. (1974). Minimal structure requirement for adjuvant activity of bacterial peptidoglycan derivatives. Biochemical and Biophysical Research Communications 59, 1317-1325.

FrYER, H. C. (1966). Fisher's test of least significant difference. In Concepts and Methods of Experimental Statistics, p. 260. Boston: Allyn \& Bacon.

GORDEE, R. S. \& SIMPSON, P. J. (1967): Relationships of $\mathrm{X}$ irradiation to the enhancement of Candida albicans infection. Journal of Bacteriology 94, 6-12.

Gotze, O. \& Muller-Eberhard, H. J. (1971). The C3-activator system; an alternate pathway of complement activation. Journal of Experimental Medicine 134, 90s-113s.

Howard, L. G., Biozzi, G., Halpern, B. N., Stiffel, C. C. \& Mouton, D. (1959). The effect of Mycobacterium tuberculosis (BCG) infection on the resistance of mice to bacterial endotoxin and Salmonella enteritidis infection. British Journal of Experimental Pathology 40, 281-291.

IsHII, S. (1975). The Fisher's test of direct probability.
In Textbook of Statistics for Biological Experiments, pp. 107-109. Tokyo: Baifukan.

Ishikawa, H., NiInobe, S. \& Tsurufuji, S. (1968). Studies of the mode of action of anti-inflammatory agents. I. Quantitative analysis of anti-inflammatory effect by carboxymethyl cellulose pouch method. Yakugaku Zasshi 88, 1472-1477.

Kaplan, H. S., Speck, R. S. \& Jawetz, E. (1952). Impairment of antimicrobial defenses following total body irradiation of mice. Journal of Laboratory and Clinical Medicine 40, 682-691.

LANDY, M. (1956). Increase in resistance following administration of bacterial lipopolysaccharides. Annals of the New York Academy of Sciences 66, 292-303.

Matsumoto, K., Ogawa, H., Kusama, T., Nagase, O., Sawaki, N., Inage, M., Kusumoto, S., Shiba, T. \& AzUMA, I. (1981). Stimulation of nonspecific resistance to infection induced by 6-O-acyl muramyl dipeptide analogs in mice. Infection and Immunity 32, 748-758.

Parant, M., Parant, F., Chedid, L. \& Le Minor, L. (1975). Immunostimulants bactériens et protection de la souris infectée par Klebsiella pneumoniae résistante aux antibiotiques par mutation ou par transfert de plasmides. Annals de Immunologie 126, 319-326.

Parant, M., Parant, F., Chedid, L., Drapier, J. C., ReTITT, J. F., WiEZERBIN, J. \& Lederer, E. (1977). Enhancement of nonspecific immunity to bacterial infection by cord factor (6,6'-trehalose dimycolate). Journal of Infectious Diseases 135, 771-777.

Parant, M., Parant, F. \& Chedid, L. (1978). Enhancement of the neonate's nonspecific immunity to Klebsiella infection by muramyl-dipeptide, a synthetic immunoadjuvant. Proceedings of the National Academy of Sciences of the United States of America 75, 3395-3399.

Pennington, J. E. (1977a). Quantitative effects of immunosuppression on bronchoalveolar cells. Journal of Infectious Diseases 136, 127-131.

Pennington, J. E. $(1977 b)$. Bronchoalveolar cell response to bacterial challenge in the immunosuppressed lung. American Review of Respiratory Disease 116, 885-893.

Peters, W. P., Holland, J. F., Senn, H., Rhomberg, W. \& BANERJEE, T. (1972). Corticosteroid administration and localized leukocyte mobilization in man. New England Journal of Medicine 282, 342345.

Saslaw, S., Carlisle, H. N. \& Moheimant, M. (1972). Effect of vincristine sulfate on Pseudomonas infections in monkeys. Infection and Immunity 6, 149-155.

Sharbaugh, R. J. \& Grogan, J. B. (1969). Effect of cyclophosphamide on experimental Staphylococcus infection in the rat. Nature, London 224, 809-810.

Sickles, E. A., Greene, W. H. \& Wiernik, P. H. (1975). Clinical presentation of infection in granulocytopenic patients. Archives of Internal Medicine 135, $715-719$.

Tatsukawa, K., Mitsuyama, M., Takeya, K. \& Noмото, K. (1979). Differing contribution of polymorphonuclear cells and macrophages to protection of mice against Listeria monocytogenes and Pseudomonas aeruginosa. Journal of General Microbiology 115, 161-166. 
Tripathy, S. P. \& Mackaness, G. B. (1969). The effect of cytotoxic agents on the primary immune response to Listeria monocytogenes. Journal of Experimental Medicine 130, 1-16.

WARD, P. A. (1966). The chemosuppression of chemotaxis. Journal of Experimental Medicine 124, 209-229.
Young, L. S. \& ARMSTrong, D. (1972). Human immunity to Pseudomonas aeruginosa. I. In vitro interaction of bacteria, polymorphonuclear leukocyte, and serum factors. Journal of Infectious Diseases 126, 257-276. 